\title{
Celastrol and an EGCG pro-drug exhibit potent chemosensitizing activity in human leukemia cells
}

\author{
ANDREW DAVENPORT ${ }^{1 *}$, MICHAEL FREZZA $^{1 *}$, MIN SHEN $^{1}$, YUBIN GE $^{2}$, \\ CONGDE HUO $^{3}$, TAK HANG CHAN ${ }^{3}$ and Q. PING DOU ${ }^{1}$
}

\author{
${ }^{1}$ The Prevention Program, Barbara Ann Karmanos Cancer Institute, Department of Pathology; ${ }^{2}$ Developmental Therapeutics \\ Program, Barbara Ann Karmanos Cancer Institute, Department of Pediatrics, Wayne State University School of \\ Medicine, Detroit, MI, USA; ${ }^{3}$ Department of Chemistry, McGill University, Montreal, Quebec, Canada
}

Received October 8, 2009; Accepted November 13, 2009

DOI: 10.3892/ijmm_00000366

\begin{abstract}
Chemotherapy remains the staple of treatment for many types of leukemia. Despite the positive impact on extending overall survival in patients with hematological malignancies, new treatment strategies are needed to reduce the nonspecific toxicity and improve the efficacy of treatment. Celastrol, derived from the 'Thunder God Vine' and Pro-EGCG, a pre-drug version of green tea polyphenol EGCG have shown potent biological activity in vitro and in vivo. Whether these natural products augment the efficacy of conventional chemotherapy in the treatment of leukemia cells has yet to be demonstrated. Here we demonstrate that these natural products could sensitize the effect of chemotherapy in both K-562 and Jurkat $\mathrm{T}$ human leukemia cells. Accordingly, this potent biological activity was associated with increased levels of leukemia cell killing, caspase 3 activation, and poly(ADPribose) polymerase cleavage. Furthermore, the higher levels of apoptotic indices were associated with decreased levels of Bcr-Abl oncoprotein in K-562 cells. Taken together, our findings present a compelling rationale for the development of combination strategies using natural products in the treatment of hematological malignancies.
\end{abstract}

\section{Introduction}

Chemotherapy and radiation remain at the forefront of treatment options for many patients afflicted with hematological malignancies. Daunorubicin, derived from the anthracycline family is a commonly used cytotoxic agent in the treatment

Correspondence to: Dr Q. Ping Dou, The Prevention Program, Barbara Ann Karmanos Cancer Institute, Department of Pathology, Wayne State University School of Medicine, 4100 John R Road, Detroit, MI 48201, USA

E-mail: doup@karmanos.org

*Contributed equally

Key words: natural products, pro-drug, chemosensitizing, chemotherapy, apoptosis, leukemia of various cancers including leukemia (1). It has been widely reported that Daunorubicin exerts its activity by preventing DNA replication and inhibiting transcription (2). Cytarabine, otherwise known as Ara-C, is a widely employed chemotherapeutic agent for the treatment of various types of leukemia (3). One mode of action of Ara-C is due to its rapid conversion to triphosphate derivative (Ara-CTP), which incorporates into DNA resulting in DNA chain termination and $S$ phase arrest. Other modes of action consist of inhibition of DNA and RNA polymerases required for DNA synthesis (4). Despite the positive clinical impact of Ara-C, the collateral damage to normal cells and acquisition of drug resistance are limiting factors that impede a favorable clinical outcome (5). Therefore, new treatment strategies are warranted that can reduce nonspecific toxicity, overcome drug resistance and improve the overall efficacy of treatment.

Epidemiological studies have indicated that green tea consumption is associated with cancer-preventative effects $(6,7)$. Among the polyphenols of green tea, (-)-EGCG is the most abundant and biologically active constituent with respect to anticancer activity in several types of human cancer $(8,9)$. Various mechanisms underlying its anticancer effects have been described, including proteasome inhibition (10). However, (-)-EGCG is relatively unstable under neutral or alkaline conditions. In an attempt to enhance the stability of (-)-EGCG, peracetate-protecting groups were introduced to the reactive hydroxyls to form Pro-EGCG (11).

Celastrol is a natural compound extracted from Tripterygium wilfordii Hook $F$ and has been used for medicinal purposes in China for hundreds of years (12). Due to its anti-inflammatory effects, Celastrol has been effectively used in the treatment of various diseases $(13,14)$. Within physiological concentrations, Celastrol inhibits cancer cell proliferation and induces cell death in a broad range of cancer lines including, leukemia cells $(15,16)$. Additionally our laboratory has reported that proteasome inhibition induced by Celastrol is one mechanism responsible for its potent biological activity (17).

In this study we report that Celastrol and Pro-EGCG can sensitize two different leukemia cell lines to conventional chemotherapy. K-562 and Jurkat T leukemia cells showed increase cell killing activity when incubated with both natural compound (Celastrol or Pro-EGCG) and chemotherapy compared to natural compound or chemotherapy alone. 
A

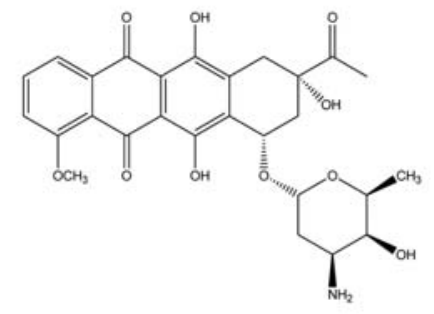

Daunorubicin (DNR)

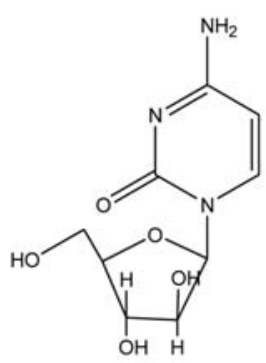

Cytarabine (Ara-C)

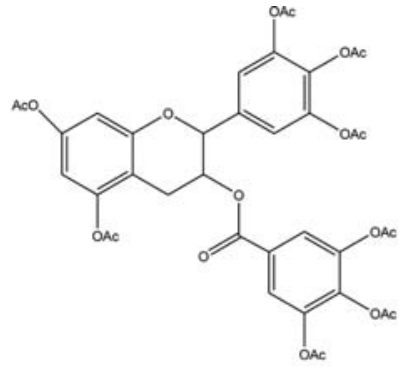

Pro-EGCG

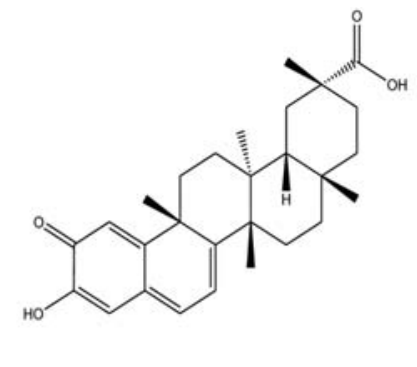

Celastrol
B

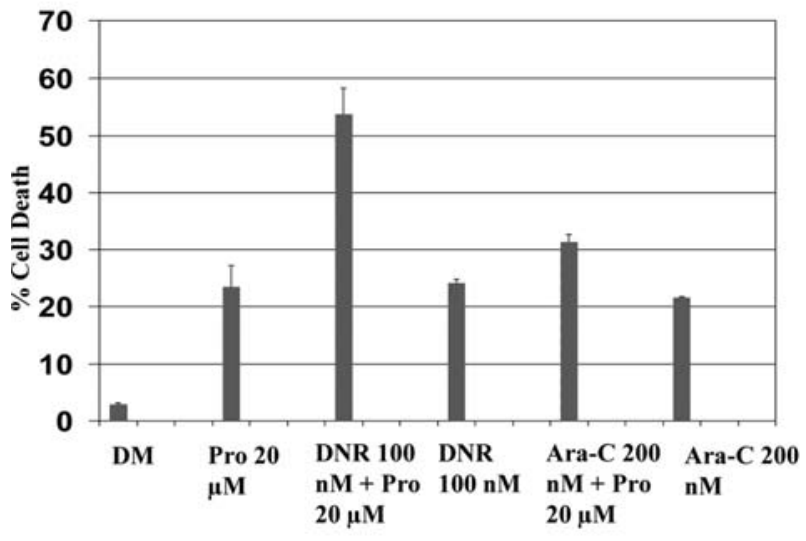

C

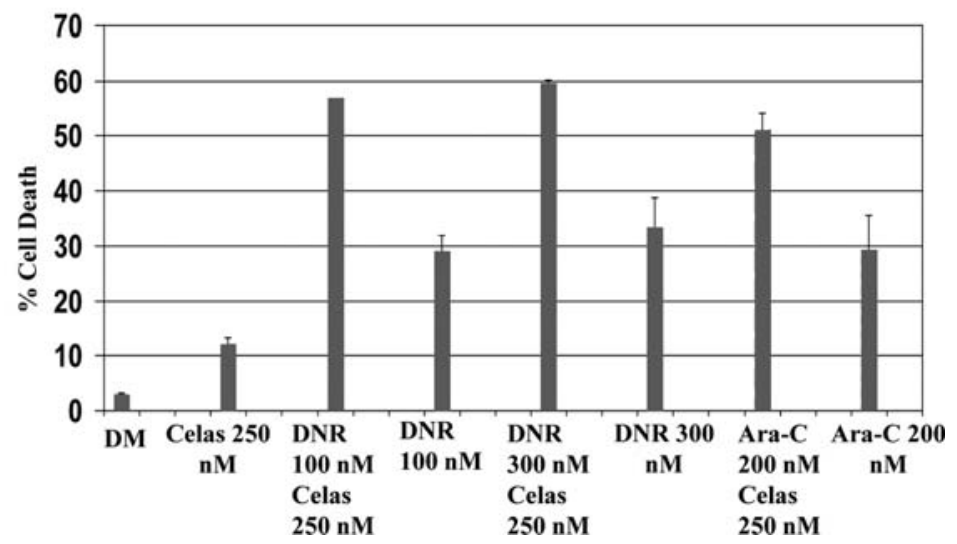

Figure 1. Cytotoxicity profile of natural products as chemosensitizing agents in K-562 cells. (A) Chemical structures of chemotherapeutic agents Daunorubicin (DNR) and cytarabine (Ara-C), and natural products Pro-EGCG (Pro) and Celastrol (Celas). (B) K-562 cells were treated with either $20 \mu \mathrm{M}$ Pro-EGCG (Pro), 100 nM DNR, $200 \mathrm{nM}$ Ara-C alone, or in combination for $72 \mathrm{~h}$, followed by trypan blue exclusion assay. (C) K-562 cells were treated with $250 \mathrm{nM} \mathrm{Celastrol} \mathrm{(Celas),}$ $100 \mathrm{nM}$ DNR, $200 \mathrm{nM}$ Ara-C alone, or in combination for $72 \mathrm{~h}$, followed by trypan blue exclusion assay. The numbers given are percentages of nonviable cells to total cells.

Consistent with increased cell killing, higher levels of caspase 3 activity and PARP cleavage and decreased levels of Bcr-abl protein (in K-562 cells) were observed when treated in combination. Our results show that natural compounds have the ability to sensitize human leukemia cells to chemotherapy and provide evidence for the rational design of new combination treatments with less toxic agents, such as natural products in the treatment of hematological malignancies.

\section{Materials and methods}

Materials. Human Jurkat (T-cell leukemia) and K-562 (CML at blast crisis) cells were purchased from ATCC (Manassas, VA). Purified Celastrol ( $>98 \%)$ was purchased from Calbiochem, Inc., (San Diego, CA). Synthesis of Pro-EGCG from (-)-EGCG was done as previously described $(11,18)$. Daunorubicin, Cytarabine (Ara-C), Dimethylsulfoxide (DMSO) and trypan blue dye were purchased from Sigma-Aldrich (St. Louis, MO). RPMI 1640, penicillin, and streptomycin were from Invitrogen (Carlsbad, CA). Fetal bovine serum was purchased from Aleken Biologicals (Texarkana, AK). Mouse monoclonal antibody against PARP was from Biomol International (Plymouth Meeting, PA). Mouse monoclonal antibody to $\mathrm{Abl}$, and rabbit polyclonal to Bcr were purchased from Santa Cruz Biotechnology (Santa Cruz, CA). Anti-mouse and antigoat secondary antibodies were from BioRad (Hercules, CA).
HyGLO chemiluminescent reagent was purchased from Denville Scientific (Metuchen, NJ).

Cell cultures and whole cell extract preparation. Jurkat $\mathrm{T}$ and K-562 cells were cultured in RPMI-1640 medium, supplemented with $10 \%$ fetal bovine serum and penicillin/ streptomycin, and maintained in a humidified incubator at $37^{\circ} \mathrm{C}$ and $5 \% \mathrm{CO}_{2}$. A whole-cell extract was prepared as previously described (19).

Trypan blue assay. The trypan blue dye exclusion assay was performed by mixing $100 \mu 1$ of cell suspension with $50 \mu 1$ trypan blue dye before injecting into a hemocytometer and counting. The number of cells that absorbed the dye and those that excluded the dye were counted, from which the percentage of nonviable cell number to total cell number was calculated.

Caspase 3 activity assay. Leukemia cells were treated with each drug or combination at indicated concentrations for $72 \mathrm{~h}$, harvested and lysed. The substrate Ac-DEVD-AMC ( $40 \mu \mathrm{mol} / \mathrm{l})$ was then incubated with the prepared cell lysates for $24 \mathrm{~h}$, followed by measurement of caspase 3 activity as previously described (20).

Western blot analysis. Human leukemia cells were treated, harvested, and lysed. Cell lysates (40-50 $\mu \mathrm{g})$ were separated 
by SDS-PAGE and transferred to a nitrocellulose membrane, followed by visualization using the HyGLO reagent (Denville Scientific; Metuchen, NJ).

\section{Results}

Celastrol and Pro-EGCG sensitize K-562 human leukemic cells to chemotherapy. Celastrol and Pro-EGCG (Fig. 1A) have been shown to possess potent antitumor activity in vitro and in vivo, including growth suppression and apoptosis induction $(17,21)$. Whether these non-toxic agents sensitize leukemia cells to chemotherapy has yet to be reported. These natural compounds were first tested for their ability to enhance the activity of chemotherapeutic agents, Ara-C and Daunorubicin (Fig. 1A) toward human leukemia cells by measuring cell death induction in a trypan blue assay. The procedure was undertaken by treating K-562 cells with natural compounds, clinically relevant doses of Ara-C or Daunorubicin, or combination of natural compound and chemotherapy for $72 \mathrm{~h}$, followed by measurement of non-viable cells. When $20 \mu \mathrm{M}$ Pro-EGCG was combined with $100 \mathrm{nM}$ Daunorubicin, 53\% cell death was achieved (Fig. 1B). This observation is significant since only 22 and $23 \%$ cell death was achieved when incubated with Pro-EGCG or Danuorubicin alone under the same experimental conditions (Fig. 1B). Additionally, when $20 \mu \mathrm{M}$ Pro-EGCG was combined with $200 \mathrm{nM}$ Ara-C, cell death induction was increased to $\sim 31 \%$ compared to 22 and $21 \%$ when either of these agents was used alone (Fig. 1B). It is notable to point that when the unprotected parent compound (-)-EGCG was used no significant cell death was observed either alone or in combination with chemotherapy (data not shown), which was probably due to its low bioavailability.

To test whether the natural compound Celastrol could sensitize K-562 cells to chemotherapy, a similar approach was taken by measuring cell death in a trypan blue exclusion assay. Interestingly, we found when $250 \mathrm{nM}$ Celastrol was combined with $100 \mathrm{nM}$ Daunorubicin, 57\% cell death was achieved (Fig. 1C). This result was significant, since cells treated with either Celastrol or Daunorubicin alone was only able to impart $\sim 12$ and $28 \%$ cell death, respectively (Fig. 1C). Additionally when increasing the concentration of Daunorubicin to $300 \mathrm{nM}$ and using the same dose of Celastrol, cell death was increased to $\sim 60 \%$ in combination, while Daunorubicin treatment alone induced $\sim 32 \%$ cell death (Fig. 1C). When investigating the sensitizing effect of $250 \mathrm{nM}$ Celastrol with $200 \mathrm{nM}$ Ara-C, $\sim 51 \%$ cell death was achieved compared to $29 \%$ cell death when Ara-C was treated alone (Fig. 1C). These results clearly show that natural compounds Celastrol and Pro-EGCG are able to sensitize leukemic cells to chemotherapy resulting in increase cytotoxicity. However, whether these interesting results illustrate an additive or synergistic effect is rather speculative at this point and premature.

Chemosensitization by natural compounds is associated with apoptosis induction and decreased Bcr-Abl expression in $K-562$ human leukemic cells. We next set out to investigate whether apoptosis is responsible for chemosensitization by these natural compounds by measuring caspase 3 activity, apoptosis-specific PARP cleavage and $\mathrm{Bcr}-\mathrm{Abl}$ protein expression. Caspase 3 is a critical determinant in the initiation of apoptosis and is associated with the cleavage of many critical
A

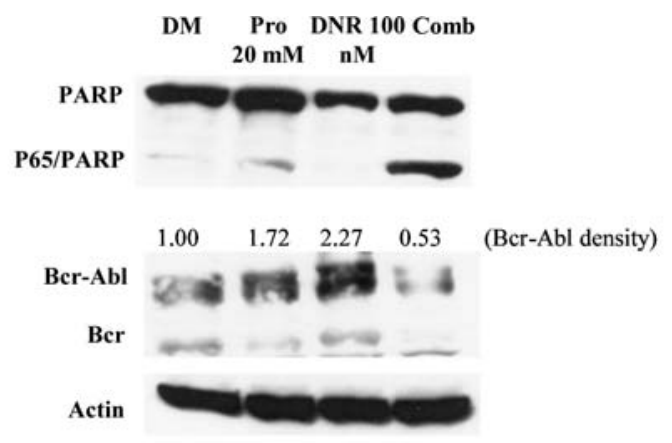

B

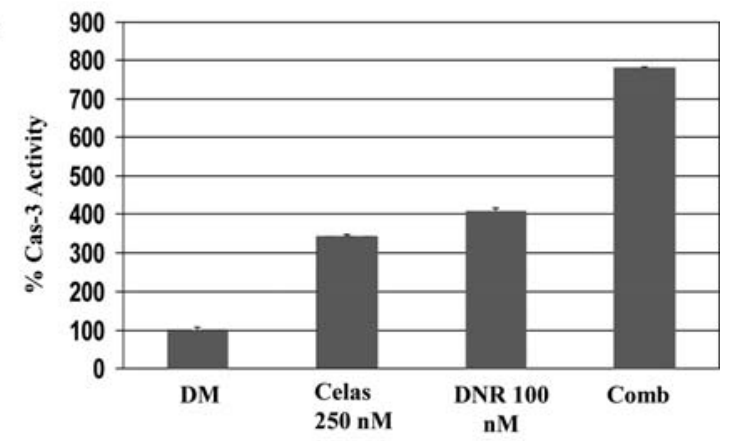

C
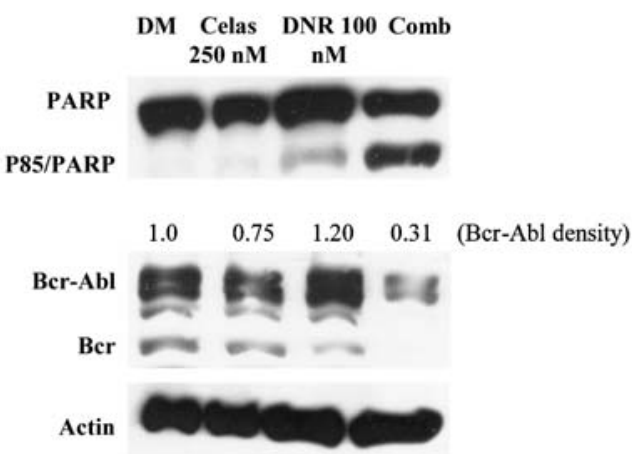

Figure 2. Induction of K-562 cell apoptosis by combination treatment of natural compounds and chemotherapy. (A) K-562 cells were treated with either DMSO control, Pro-EGCG (Pro) at $20 \mu \mathrm{M}$, Daunorubicin (DNR) at $100 \mathrm{nM}$, or combination for $72 \mathrm{~h}$, followed by measurement of PARP cleavage and expression of Bcr-Abl protein levels. (B-C) K-562 cells were treated with either DMSO control, Celastrol (Celas) at $250 \mathrm{nM}$, Daunorubicin (DNR) at $100 \mathrm{nM}$ or in combination, followed by measurement of caspase 3 activity (B) and PARP cleavage and expression levels of Bcr-Abl protein in Western blot analysis (C). Actin was used as a loading control.

cellular substrates, including poly(ADP-ribose) polymerase or PARP (22). K-562 cells were first treated with either $20 \mu \mathrm{M}$ Pro-EGCG, $100 \mathrm{nM}$ Daunorubicin, or combination of the two for $72 \mathrm{~h}$, and their cell lysates were measured for caspase 3 activity and PARP cleavage. Our results show when both agents were used in combination, caspase 3 activity increased more significantly compared to each treatment alone and DMSO solvent control (data not shown). Consistent with higher levels of caspase 3 activity in co-treated cells, significantly higher levels of PARP cleavage was observed, compared to either agent treated alone (Fig. 2A). In another experiment, combining $250 \mathrm{nM}$ celastrol with $100 \mathrm{nM}$ Daunorubicin elicited 7.8-fold increase in caspase 3 activity, compared to only 3.4- or 4.0-fold increase for either treatment alone (Fig. 2B). This notable increase in caspase 3 activity 


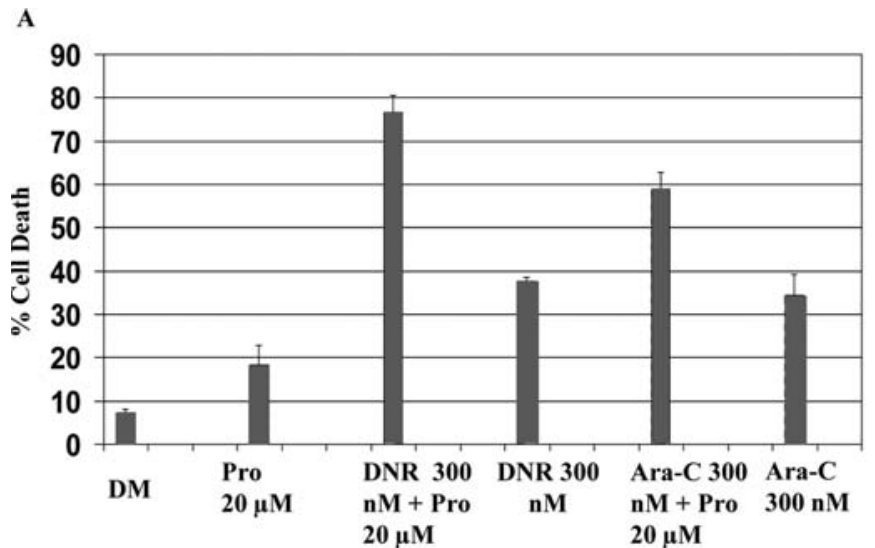

C

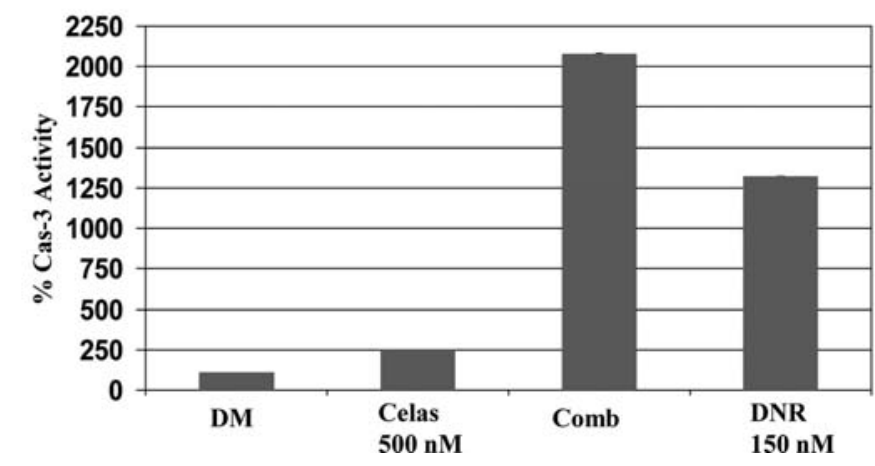

B

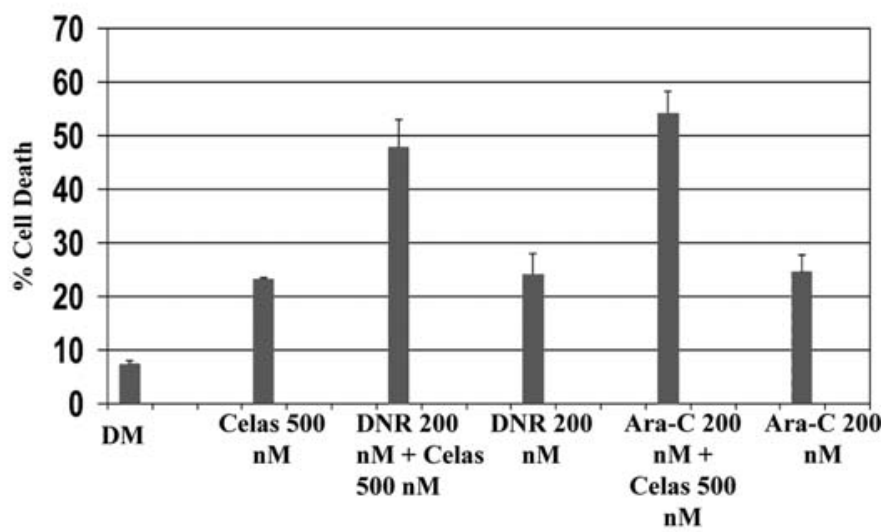

D

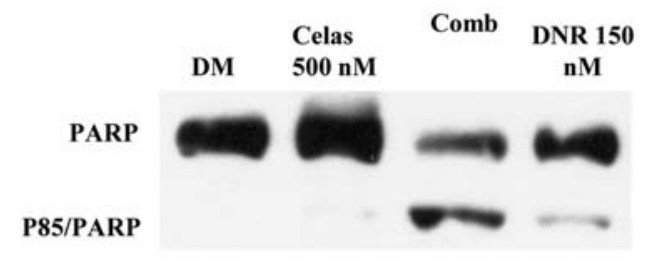

Figure 3. Combination treatment of natural compounds and chemotherapy was associated with higher cell killing and indices of apoptosis in Jurkat $\mathrm{T}$ cells. (A) Jurkat T cells were treated with either DMSO control, Pro-EGCG (Pro) at $20 \mu \mathrm{M}$, DNR at $300 \mathrm{nM}$ or Ara-C at $300 \mathrm{nM}$, or combination for $72 \mathrm{~h}$, followed by trypan blue exclusion assay. (B) Jurkat T cells were treated with Celastrol (Celas) at $500 \mathrm{nM}$, DNR at $200 \mathrm{nM}$, Ara-C at $200 \mathrm{nM}$ or in combination for $72 \mathrm{~h}$, followed by trypan blue assay. The numbers given are percentages of nonviable cells to total cells. (C-D) Jurkat T cells were treated with either Celastrol (Celas) at $500 \mathrm{nM}$, Daunorubicin at $150 \mathrm{nM}$, or in combination for $72 \mathrm{~h}$, followed by measurement of caspase 3 activity (C) and PARP cleavage by Western blot analysis (D). Actin was used as loading control.

from the combination treatment was accompanied by significant levels of PARP cleavage (Fig. 2C). We next tested whether the higher apoptosis indices found in combination treatment is associated with decreased levels of Bcr-Abl protein in CMLderived K-562 cells. This molecular abnormality functions as a constitutively active receptor tyrosine kinase and represents the underlying pathologic event in the formation of CML (23). Interestingly, when K-562 cells were co-treated with Pro-EGCG and Daunorubicin, we found $~ 50 \%$ decrease in Bcr-Abl protein levels (Fig. 2A). Additionally, when cells were treated with combination of Celastol and Daunorubicin, we found $\sim 70 \%$ decrease in Bcr-abl protein levels (Fig. 2C). These results show that chemosensitization of K-562 cells by natural compounds is associated with decreased levels of Bcr-Abl protein.

Chemosensitization of human Jurkat T leukemic cells by natural compounds is associated with cell killing and apoptosis induction. After we showed the ability of natural compounds to sensitize CML-derived K-562 cells to chemotherapy, we next investigated whether this effect is consistent against human Jurkat $\mathrm{T}$ cells. We first treated Jurkat $\mathrm{T}$ cells with $20 \mu \mathrm{M}$ Pro-EGCG, $300 \mathrm{nM}$ Daunorubicin or Ara-C, or in combination for $72 \mathrm{~h}$, followed by measurement of nonviable cells. Our results show that treatment with Pro-EGCG alone only induced cell killing by $\sim 18 \%$, but when combined with Daunorubicin, cytotoxicity reached $\sim 76 \%$ (Fig. 3A). In comparison, treatment with Daunorubicin alone only reached $\sim 38 \%$ cell death (Fig. 3A). Additionally, when Jurkat cells were treated in combination with Pro-EGCG and Ara-c, cell death reached $\sim 59 \%$ compared to only $\sim 33 \%$ cell death when treated with Ara-C alone (Fig. 3A).

We next tested the chemosensitizing effect of Celastrol toward chemotherapy in Jurkat $\mathrm{T}$ cells by measuring the number of nonviable cells. Cells were treated with Celastrol at $500 \mathrm{nM}$ with or without Daunorubicin or Ara-C at $200 \mathrm{nM}$ for $72 \mathrm{~h}$, followed by the measurement of nonviable cells. Our results show that when Celastrol or Daunorubicin were used as single agents, cell killing only reached 23 and $24 \%$, respectively (Fig. 3B). However, in combination treatment, their ability to induce cell death reached $\sim 47 \%$ (Fig. 3B). Similarly, when Celastrol was used in combination with Ara-C, cell death was significantly increased to $\sim 53 \%$ (Fig 3B). In comparison, when Celastrol and Ara-C were used singularly, cytotoxicity only reached $\sim 23$ and $24 \%$, respectively (Fig. 3B).

To determine whether this enhanced cell killing by combination treatment is associated with apoptosis, caspase 3 activity and apoptosis-specific PARP cleavage was measured. Jurkat cells were treated with either $500 \mathrm{nM}$ Celastrol, $150 \mathrm{nM}$ Daunorubicin, or combination followed by the measurement 
of caspase 3 activity and PARP cleavage. Our results show that treatment with Celastrol and Daunorubicin alone induced 2.5- and 13-fold caspase 3 activity, respectively, compared to DMSO control (Fig. 3C). However, when both agents were treated in combination, caspase activity increased by $\sim 21$-fold (Fig. 3C). In addition to the significantly higher levels of caspase 3 levels, apoptosis-specific PARP cleavage was much more apparent when both agents were treated in combination (Fig. 3D). As demonstrated with K-562 cells, Pro-EGCG and Celastrol have significant ability to sensitize Jurkat $\mathrm{T}$ cells to conventional chemotherapy, as illustrated by their potent cell killing activity leading to higher levels of caspase 3 activities and PARP cleavage.

\section{Discussion}

The use of chemotherapy remains one of the important approaches among cancer therapies. Unfortunately, in many malignancies, acquisition of drug resistance and toxicity during chemotherapy constitutes a major hurdle and challenge in cancer therapy (24). Therefore, combination strategies that include natural compounds that sensitize tumor cells to chemotherapy may represent a more promising approach to cancer therapy. Emerging evidence has shown that some natural compounds, including Pro-EGCG and Celastrol have potent growth inhibitory and apoptosis-inducing effects on cultured human cancer cells and xenografts $(17,21)$. Therefore, these non-toxic agents derived from natural resources could be useful in combination with conventional chemotherapeutic agents for the treatment of human malignancies, including leukemia. In fact, increasing evidence from pre-clinical in vitro and in vivo studies have shown some success in support of conventional chemotherapeutic agents in combination with natural products $(25,26)$.

Our laboratory has previously shown that both Pro-EGCG and Celastrol exhibit potent growth suppression and apoptosis inducing effects, and that the proteasome is a critical target underlying their biological effects $(17,21)$. However, whether these natural compounds could sensitize the effect of conventional chemotherapy in human leukemia cells has yet to be demonstrated. In this study, we investigated the chemosensitivity of Pro-EGCG and Celastrol toward two human leukemia cell lines, namely CML-derived K-562 cells and Jurkat T-cell leukemia cells. Interestingly, we found that both Celastrol and Pro-EGCG were able to sensitize both leukemia cell lines to the effects of chemotherapy, resulting in a significant increase in cell killing (Figs. 1-3). Our data also show that both natural compounds are much more effective in sensitizing leukemia cells to Daunorubicin compared to Ara-C (Fig. 1B,C; Fig. 2A). It is important to point out that whether this chemosensitizing effect is the result of an additive or synergistic effect is rather speculative and further investigation is needed in order to establish a firm conclusion.

An important aspect of the current study was to determine whether chemosensitization by natural products is associated with apoptosis induction and decreased levels of Bcr-Abl in leukemia cells. Bcr-abl is a constitutively active kinase that represents a critical molecular determinant in the development of CML (23). Our results show that associated with increased cell killing, both Pro-EGCG and Celastrol could sensitize $\mathrm{K}-562$ cells to Daunorubin as shown by higher levels of caspase 3 activity and PARP cleavage (Fig. 2B,C). Additionally, associated with the higher apoptotic indices in combination treatment were decreased levels of Bcr-Abl protein (Fig. 2A,C). This represents a significant achievement since many CML patients become refractory to TKI therapy and eventually relapse upon cessation of treatment (27). Furthermore, higher levels of PARP cleavage were apparent in Jurkat T cells when Celastrol is used in combination with daunorubicin, but at higher concentrations (Fig. 3D).

While the precise mechanism responsible for chemosensitization by natural compounds is not clearly understood, our current results and previous studies validate the need for new combination strategies that incorporate natural compounds as chemosensitizing agents in the treatment of hematological malignancies.

\section{Acknowledgements}

This work was partially supported by the National Cancer Institute (Grant Number, 1R01CA120009 and 3R01CA12000904S1). M.F. acknowledges a training grant from the National Cancer Institute (T32-CA009531) and A.D. acknowledges an Undergraduate Summer Fellowship in Cancer Research Program, Karmanos Cancer Institute. Financial support from NSERC of Canada is gratefully acknowledged.

\section{References}

1. Tallman MS, Gilliland DG and Rowe JM: Drug therapy for acute myeloid leukemia. Blood 106: 1154-1163, 2005.

2. Huang RS, Duan S, Kistner EO, Bleibel WK, Delaney SM, Fackenthal DL, Das S and Dolan ME: Genetic variants contributing to daunorubicin-induced cytotoxicity. Cancer Res 68: 3161-3168, 2008.

3. Liu J, Mi Y, Fu M, Yu W, Wang Y, Lin D, Bian S and Wang J: Intensive induction chemotherapy with regimen containing intermediate dose cytarabine in the treatment of de novo acute myeloid leukemia. Am J Hematol 84: 422-427, 2009.

4. Hubeek I, Stam RW, Peters GJ, Broekhuizen R, Meijerink JP, van Wering ER, Gibson BE, Creutzig U, Zwaan CM, Cloos J, Kuik DJ, Pieteres R and Kaspers GJ: The human equilibrative nucleoside transporter 1 mediates in vitro cytarabine sensitivity in childhood acute myeloid leukemia. Br J Cancer 93: 1388-1394, 2005.

5. Karbasian-Esfahani M, Wiernik PH, Novik Y, Paietta E and Dutcher JP: Indarubicin and standard dose cytosine arabinoside in adults with recurrent and refractory acute lymphocytic leukemia. Cancer 101: 1414-1419, 2004.

6. Fujiki H, Suganuma M, Imai K and Nakachi K: Green tea: cancer preventative beverage and/or drug. Cancer Lett 188: 9$13,2002$.

7. Inoue M, Tajima K, Hirose $\mathrm{K}$, et al: Tea and coffee consumption and the risk of digestive tract cancers: data from a comparative case-referent study in Japan. Cancer Causes Control 9: 209-216, 1998.

8. Shanafelt TD, Lee YK, Call TG, et al: Clinical effects of oral green tea extract in four patients with low grade B-cell malignancies. Leuk Res 30: 707-712, 2006.

9. Sartippour MR, Heber D, Ma J, Lu Q, Go VL and Nguyen M: Green tea and its cathechins inhibit breast cancer xenografts. Nutr Cancer 40: 149-156, 2001.

10. Nam S, Smith DM and Dou QP: Inhibition of proteasome activity in vitro and in vivo by ester bond-containing tea polyphenols. $\mathrm{J}$ Biol Chem 276: 13322-13330, 2001.

11. Lam WH, Kazi A, Kuhn DJ, et al: A potential prodrug for a green tea polyphenol proteasome inhibitor: evaluation of the peracetate ester of (-) epigallocatechin gallate [(-)-EGCG]: Bioorg Med Chem 12: 5587-5593, 2004. 
12. Setty AR and Sigal LH: Herbal medications commonly used in the practice of rheumatology: mechanisms of action, efficacy, and side effects. Semin Arthritis Rheum 34: 773-784, 2005.

13. Tao X, Younger J, Fan FZ, Wang B and Lipsky PE. Benefit of an extract of Tripterygium wilfordii Hook $F$ in patients with rheumatoid arthritis: a double-blind, placebo-controlled study. Arthritis Rheum 46: 735-743, 2002.

14. Pinna GF, Fiorucci M, Reimund JM, Taquet N, Arondel Y and Muller CD: Celastrol inhibits pro-inflammatory cytokine secretion in Crohn's disease biopsies. Biochem Biophys Res Commun 322: 778-786, 2004.

15. Chang FR, Hayashi K, Chen LH, Liaw CC, Bastow KF, Nakanishi Y, Nozaki H, Cragg GM, Wu YC and Lee KH: Antitumor agents. 228. five new agarofurans, Reissantins A-E, and cytotoxic principles from Reissantia buchananii. J Nat Prod 66: 1416-1420, 2003.

16. Nagase M, Oto J, Sugiyama S, Yube K, Takaishi Y and Sakato N: Apoptosis induction in HL-60 cells and inhibition of topoisomerase II by triterpene celastrol. Biosci Biotechnol Biochem 67: 1883-1887, 2003.

17. Yang H, Chen D, Cui QC, Yuan X and Dou QP: Celastrol, a triterpene extracted from the Chinese 'Thunder of God Vine' is a potent proteasome inhibitior and suppresses human prostate cancer growth in nude mice. Cancer Res 66: 4758-4765, 2006.

18. Wan SB, Dou QP and Chan TH: Regiospecific and enantioselective synthesis of methylated metabolites of tea catechins: Tetrahedron 62: 5897-5904, 2006.
19. An B and Dou QP: Cleavage of retinoblastoma protein during apoptosis: an interleukin $1 \beta$-converting enzyme-like protease as candidate. Cancer Res 56: 438-432, 1996.

20. An B, Goldfarb RH, Siman R and Dou QP: Novel dipeptidyl proteasome inhibitiors overcome $\mathrm{Bcl}-2$ protective function and selectively accumulate the cyclin-dependent kinase inhibitor p27 and induce apoptosis in transformed, but not normal, human fibroblasts: Cell Death Differ 5: 1062-1075, 1998.

21. Landis-Piwowar KR, Huo C, Chen D, Milacic V, Shi G, Chan TH and Dou QP: A novel prodrug of the green tea polyphenol(-)epigallocatechin-3-gallate as a potential anticancer agent. Cancer Res 67: 4303-4310, 2007.

22. Nicholson DW and Thornberry NA: Apoptosis. Life and death decisions. Science 299: 214-215, 2003

23. Savona M and Talpaz M: Getting to the stem of chronic myeloid leukemia. Nature Rev 8: 341-350, 2008.

24. Sarkar FH and Li YW: Harnessing the fruits of nature for the development of multi-targeted cancer therapeutics. Cancer Treat Rev 35: 567-607, 2009.

25. Sarkar FH and Li YW: Targeting multiple signal pathways by chemopreventative agents for cancer prevention and therapy. Acta Pharmacol Sin 28: 1305-1315, 2007.

26. Dhillon N, Aggarwal BB, Newman RA, et al: Phase II trial of curcumin in patients with advanced pancreatic cancer. Clin Cancer Res 14: 4491-4499, 2008.

27. Usuki K, Lijima K, Lki S and Urabe A: Cytogenetic relapse after cessation of imatinib therapy. Leuk Res 29: 237-238, 2005. 